\title{
ACTH-independent Cushing syndrome
}

INSERM

\section{Source}

INSERM. (1999). Orphanet: an online rare disease and orphan drug data base. ACTHindependent Cushing syndrome. ORPHA:99893

Adrenocorticotropic hormone (ACTH) independent Cushing syndrome is a form of endogenous Cushing syndrome (CS; see this term) that may result from excess secretion of cortisol by either a unilateral and benign (adrenocortical adenoma: 55-60\%) or malignant (adrenocortical carcinoma: 35-40 \%) adrenocortical tumor or by bilateral adrenal secretion by macronodular adrenal hyperplasia (AIMAH), as an isolated disease or as part of McCune-Albright syndrome (MAS), or by primary pig mented nodular adrenocortical disease (PPNAD), as an isolated disease or as part of Carney complex (CNC; see these terms). 